\title{
The Hugoniot and Strength of Ultem 1000 Polyetherimide
}

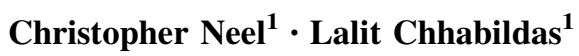

Received: 2 July 2015 / Accepted: 22 July 2015/Published online: 27 August 2015

(C) The authors 2015

\begin{abstract}
Parallel-plate impact studies using a single stage powder gun have been performed to investigate the shock and subsequent release behavior of the commercial polyetherimide polymer Ultem ${ }^{\mathrm{TM}}$ up to $13 \mathrm{GPa}$. Two different experimental configurations were used to observe both the shock and unloading behavior. In one configuration, the unloading was continuously tracked by observing transmitted wave profiles in the Ultem samples through a transparent interferometer window. In the other configuration, the unloading was inferred from observing stress wave reverberations in an elastic target plate resulting from impact by the Ultem sample. The loading behavior indicated by the two methods agreed very well and the resulting Hugoniot was represented by $\mathrm{U}_{\mathrm{S}}=2.42+1.61 * \mathrm{u}_{\mathrm{P}}$. This study also demonstrated that the plate reverberation method of following the unloading response, though not observing the continuous unloading of the sample, agrees extremely well with the unloading response recorded using continuous data obtained using interferometry windows. The results are used to build a case that the strength $\tau$ of Ultem increases with shock stress from 0.03 to 0.08 GPa over the range investigated. Furthermore, an investigation of the ratio of the release wave velocity to the shock wave velocity indicates that a transition to bulk liquid (no strength) behavior is not achieved until Hugoniot strains exceed 0.35 for amorphous polymers such as Ultem.
\end{abstract}

Keywords Ultem · Polyetherimide $\cdot$ PEI $\cdot$ Shock · Hugoniot $\cdot$ Strength

Christopher Neel

christopher.neel.1@us.af.mil

1 Air Force Research Laboratory, Munitions Directorate, Eglin Air Force Base, Florida 32542, USA

\section{Introduction}

Ultem $^{\mathrm{TM}}$ is the trade name for polyetherimide (PEI), and was developed as a high temperature structural polymer. It has since found use in military systems where it is exposed to shock loading, and so the behavior under such loading is of interest. With a few exceptions such as PMMA [1] and epoxy [2], the shock response of polymers was not widely investigated until the last two decades, when several groups began systematic investigations [3-5]. However, identifying systematic trends across classes of polymers has largely proven elusive. Some observed trends include: (a) the lack of an elastic precursor, (b) the related observation that the zero-pressure intercept of the shock speed ("c $\mathrm{c}_{0}$ " in the linear $\mathrm{U}_{\mathrm{S}}-\mathrm{u}_{\mathrm{P}}$ Hugoniot fit) is higher than the bulk sound speed measured at ambient conditions, and c) the $\sim 20-25$ GPa phase transition attributed to the disassociation of the polymer chain structure [4]. Although it has been shown that polymers do not display an elastic precursor, they do not behave as inviscid fluids and do exhibit a shear strength or viscosity resisting deformation. This has been demonstrated primarily through the use of embedded manganin (piezoresistive) stress gauges oriented in orthogonal planes with respect to the shock front to show that the stress state is not hydrostatic. Such "lateral" gauge experiments have shown that polymers are capable of sustaining shear stresses of between 0.1 and $0.8 \mathrm{GPa}$ behind a $6 \mathrm{GPa}$ shock $[3,5]$. In those studies, it was generally (but not always) found that the shear stress increased with shock stress. One study done on PMMA at low pressures using oblique-impacts with embedded velocity gauges, reported shear stress rising to about $0.1 \mathrm{GPa}$ before decreasing beyond a shock stress of $0.6 \mathrm{GPa}$ [6].

The present work adds to this library of data on polymer shock response and measures the strength in the shocked 
material by examining the hysteresis in the load-unload path of the Ultem polymer rather than measuring the stress or material motion in a direction normal to shock propagation. Two different experimental configurations and analysis methods are used to generate the unloading path, and it is shown that the two are in very good agreement. Furthermore, an analysis based on the extrapolated bulk and measured sound speeds [7] is used to infer the strength from similar experiments, and it is shown again that the results are in good agreement.

\section{Materials and Experimental Setup}

The material investigated in this work was obtained as commercial Ultem 1000 (unfilled PEI), supplied by Piedmont Plastics, in the form of a cylinder $57.15 \mathrm{~mm}$ diameter and $610 \mathrm{~mm}$ long. Discs were machined from the rod and lapped and polished. No further conditioning or treatment on the material was performed. The ambient ultrasonic sound speeds were measured and found to be $2.45 \pm 0.04$ and $1.04 \pm 0.005 \mathrm{~km} / \mathrm{s}$ for the longitudinal $\mathrm{c}_{\mathrm{L}}$ and shear $\mathrm{c}_{\mathrm{S}}$ wavespeed respectively, yielding a bulk soundspeed $c_{B}$ of $2.13 \mathrm{~km} / \mathrm{s}$ and Poisson's ratio $v$ of 0.39 . The density of each sample used in the experiments was determined using the mass with the measured dimensions and found to be $1.281 \pm 0.003 \mathrm{~g} / \mathrm{cc}$. The material was amber in color and appeared transparent, but absorption profiles and crystallinity were not determined. Due to the apparent transparency and also the manufacturer's description, we assume it is completely amorphous.

Impact experiments were performed on a single-stage, $60 \mathrm{~mm}$ bore powder gun at Eglin Air Force Base, FL [8]. Two different types of impact setups were used- hereafter referred to as the "plate-push" configuration and the "window" configuration. Both configurations are illustrated in Fig. 1 and described further below. In both configurations the projectile was faced with a lapped and polished Ultem disc, at least $46 \mathrm{~mm}$ diameter. In the plate- push experiments, the impactor was $\sim 10 \mathrm{~mm}$ thick Ultem and the target consisted of a relatively thin witness plate, composed of either sapphire or Corning 7980 fused silica with a thin coating of $\mathrm{Au} / \mathrm{Pd}$ deposited on the back surface to serve as a reflector. In the window experiments, the impactor was approximately $2.8 \mathrm{~mm}$ thick, and in most cases was backed by void to generate an unloading wave. The target was a sapphire (c-cut) disc, $44 \mathrm{~mm}$ diameter and $22 \mathrm{~mm}$ thick, faced with a thin coating of silver. The silver coated face was the impact face, and was monitored from the rear by PDV [9] and VISAR [10] interferometry probes. Additional window configuration experiments were performed using PMMA as the window material, with aluminum deposited as the reflective impact face. For both configurations, impact tilt was measured by shorting pins mounted flush with the target surface, and impact velocity was measured using either multiple PDV probes or a combination of PDV and shorting pins. The details for each experiment are given in Table 1.

\section{Data Reduction}

The data reduction analysis used for both plate-push and window experiments relies on the impedance matching method. The method has been thoroughly described elsewhere [11] but it is briefly summarized here, using Fig. 2 for the example of an Ultem impactor striking sapphire at $2.184 \mathrm{~km} / \mathrm{s}$ (shot 13-12, plate-push configuration). The compressed state 1 results from the impact of quiescent sapphire (state $\mathrm{S} 0$ in the figure) and moving, but unstressed, Ultem (state U0 in the figure). The loading wave propagates through the sapphire and reflects as an unloading wave from the rear (free) surface of the sapphire; the material behind this unloading wave is at state 2 . The velocity at the free surface is measured (by PDV or VISAR), and so the velocity corresponding to state 2 is measured and found to be $0.579 \mathrm{~km} / \mathrm{s}$ in this example. Since the plate (sapphire in this case) is elastic, the velocity
Fig. 1 Experimental setups. On the left is a schematic of the "window" (Win) experiments, and on the right is a depiction of the "plate-push" (PP) experiments
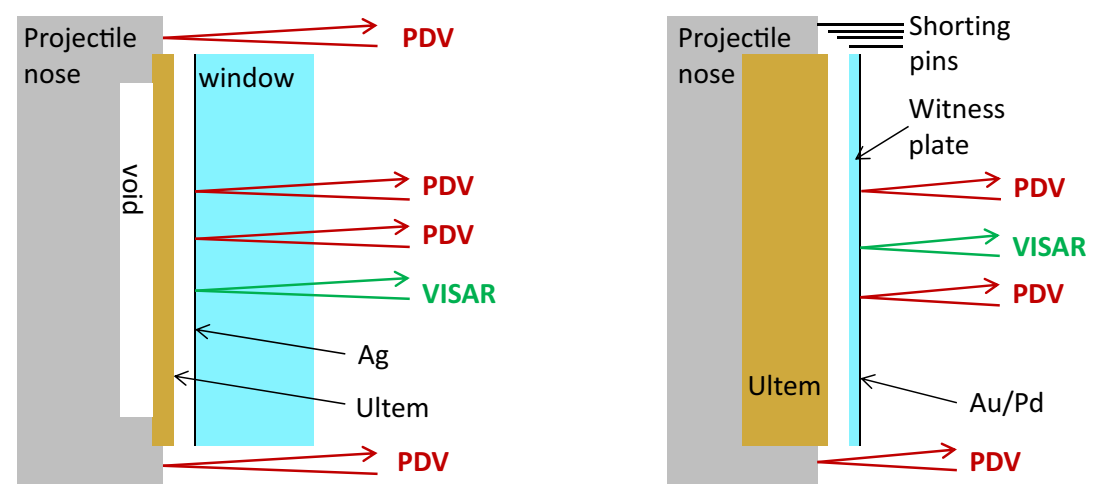
Table 1 Experimental matrix and Hugoniot data for Ultem

\begin{tabular}{|c|c|c|c|c|c|c|c|c|}
\hline Shot & Expt type & $\begin{array}{l}\text { Impactor } \\
\text { thickness }(\mathrm{mm})\end{array}$ & $\begin{array}{l}\text { Target } \\
\text { thickness }(\mathrm{mm})\end{array}$ & $\mathrm{V}_{\mathrm{imp}}(\mathrm{km} / \mathrm{s})$ & $\mathrm{V}_{\text {meas }}(\mathrm{km} / \mathrm{s})$ & $\mathrm{u}_{\mathrm{P}}(\mathrm{km} / \mathrm{s})$ & $\mathrm{U}_{\mathrm{S}}(\mathrm{km} / \mathrm{s})$ & $\mathrm{P}(\mathrm{GPa})$ \\
\hline $13-12$ & PP Sapp & 10.113 & 1.085 & 2.184 & 0.579 & 1.895 & 5.457 & 13.24 \\
\hline $13-13$ & PP Sapp & 10.112 & 1.083 & 1.528 & 0.352 & 1.353 & 4.588 & 7.97 \\
\hline $13-21$ & PP FS & 10.117 & 3.017 & 1.096 & 0.628 & 0.782 & 3.673 & 3.677 \\
\hline $13-22$ & PP FS & 10.120 & 3.021 & 0.685 & 0.346 & 0.512 & 3.209 & 2.106 \\
\hline $13-23$ & PP FS & 10.118 & 3.025 & 0.363 & 0.165 & 0.281 & 2.895 & 1.042 \\
\hline $14-04$ & Win Sapp & 2.814 & 22.01 & 2.136 & 0.281 & 1.854 & 5.416 & 12.86 \\
\hline $14-05$ & Win Sapp & 2.819 & 22.02 & 1.287 & 0.140 & 1.147 & 4.302 & 6.32 \\
\hline 30 & Win PMMA & 2.736 & 12.50 & 0.363 & 0.178 & 0.185 & 2.555 & 0.60 \\
\hline $30^{\mathrm{a}}$ & & & & & 0.180 & 0.183 & 2.615 & 0.61 \\
\hline 31 & Win PMMA & 2.789 & 12.53 & 0.665 & 0.338 & 0.327 & 2.968 & 1.24 \\
\hline 32 & Win PMMA & 2.790 & 12.46 & 0.927 & 0.475 & 0.452 & 3.226 & 1.87 \\
\hline $32^{\mathrm{a}}$ & & & & & 0.473 & 0.454 & 3.196 & 1.86 \\
\hline 33 & Win PMMA & 2.790 & 12.46 & 1.250 & 0.624 & 0.626 & 3.266 & 2.62 \\
\hline $33^{\mathrm{a}}$ & & & & & 0.628 & 0.622 & 3.314 & 2.64 \\
\hline 34 & Win PMMA & 2.789 & 12.54 & 1.477 & 0.760 & 0.717 & 3.675 & 3.38 \\
\hline 35 & Win PMMA & 2.789 & 12.65 & 1.976 & 0.993 & 0.983 & 3.834 & 4.83 \\
\hline 36 & Win PMMA & 2.789 & 12.65 & 2.223 & 1.155 & 1.068 & 4.351 & 5.95 \\
\hline 41 & Win PMMA & 2.736 & 18.89 & 2.280 & 1.170 & 1.110 & 4.265 & 6.06 \\
\hline $41^{\mathrm{a}}$ & & & & & 1.163 & 1.117 & 4.202 & 6.01 \\
\hline
\end{tabular}

${ }^{a}$ Window shots configured partially as shock-release and partially as windowed shock-reshock experiment (not shown in Fig. 1). The shockreshock points (denoted by $*$ ) are used for Hugoniot and sound speed in the shocked state determination only

$P P$ plate push configuration, Win window configuration, Sapp sapphire, FS fused silica

corresponding to state 1 is half that of state 2 . From the known behavior of the target plate and the velocity of state 1 , the magnitude of the stress $\sigma$ that brought the target plate to state 1 is known. From continuity across the impact interface, the stress $\sigma$ and velocity in the Ultem sample are identical to that in the witness plate, immediately yielding $\sigma_{1}$. Since the initial (impact) velocity of the Ultem is also known, the jump in particle velocity $\mathrm{u}_{\mathrm{P}}$ is found from $u_{P}=V_{\text {impact }}-u_{P, 1}$. This $\sigma-\mathrm{u}_{\mathrm{P}}$ data pair for Ultem constitutes the Hugoniot point for state 1. Conservation of momentum across the wavefront then yields the Ultem shock velocity as $U_{S}=\sigma /\left(\rho_{0} u_{P}\right)$.

Similarly, as the witness plate "rings up", it gradually accelerates to the impact velocity (Fig. 2) by successively loading and unloading against the Ultem, which is progressively unloading with each wave transit through the plate. The even numbered states correspond to the gradually increasing free surface velocities (resulting from stress decreased in the Ultem odd-numbered states) captured by the VISAR or PDV probes. The odd-numbered states are obtained from $u_{P, i}=\left(u_{P, i-1}+u_{P, i+1}\right) / 2$. Once all the oddnumbered $\mathrm{u}_{\mathrm{P}, \mathrm{i}}$ are calculated, the corresponding stresses $\sigma_{\mathrm{i}}$ are calculated from known properties of the witness plate. Again from continuity across an interface, the odd- numbered $\sigma_{\mathrm{i}}$ in the witness plate equals that in the Ultem sample, and so $\mathrm{u}_{\mathrm{P}, \mathrm{i}}, \sigma_{\mathrm{i}}$ data is generated corresponding to the unloading of the Ultem. This data is used later to generate the unloading paths using similar impedance matching procedures.

The impedance matching method used for the window experiments is shown in Fig. 3. It is somewhat simpler than the situation for the plate-push experiments, because in this case, the interferometer probes the interface between the materials directly (rather than the free surface of the target), and so state 1 is obtained directly and is held until unloading begins. However, for the window experiments, the unloading originates from the back surface of the impactor, rather than from the target. The resulting release fan interacts with the window until the stress across the impact interface becomes tensile and the materials separate (not shown).

\section{Results}

\section{Loading}

Impact velocities and interferometry traces were obtained for all experiments. In the plate-push experimental data 
Fig. $2 \mathrm{P}-\mathrm{u}_{\mathrm{P}}$ and corresponding Lagrangian $\mathrm{x}-\mathrm{t}$ plot illustrating impedance matching method for the plate-push method, where Ultem strikes a thin sapphire plate at $2.184 \mathrm{~km} / \mathrm{s}$. In the $\mathrm{P}-\mathrm{u}_{\mathrm{P}}$ plot the release states in Ultem are represented by the intersection points, whereas in the $\mathrm{x}-\mathrm{t}$ plot the states are regions between the characteristic lines. More complete descriptions of the method can be found elsewhere $[11,12]$ (Color figure online)
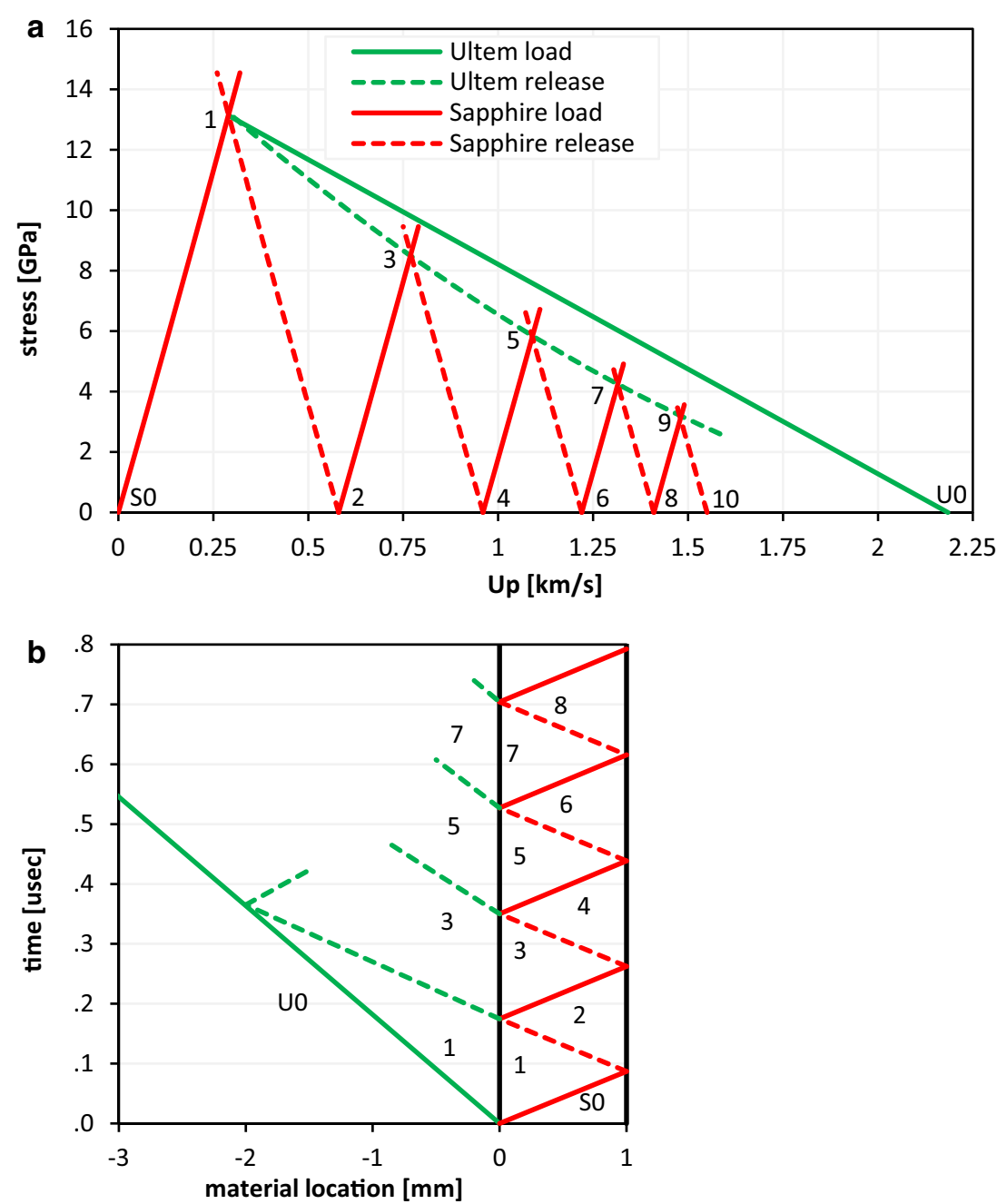

(Fig. 4), the plate is seen to ring up in steps, with each step corresponding to the loading and subsequent unloading of the witness plate after impact by the thick Ultem impactor. The first step (plateau) in each trace contains information corresponding to the loading of the Ultem, and subsequent steps correspond to a step-wise unloading of the Ultem. For the window experiments, the particle velocity traces from the Ultem/window interface are shown in Fig. 5. The velocities in this figure have been corrected for the index of refraction of sapphire and PMMA using methods and data from the literature [1, 13, 14]. From the traces in the figure, it is clear that a steady Hugoniot state is obtained, followed by a smooth release. Impact velocities and measured material velocities corresponding to the traces shown in Figs. 4 and 5 are given in Table 1.

Hugoniot points were obtained for all the experiments using standard impedance matching techniques described previously, using the following Hugoniot properties for sapphire, PMMA, and fused silica. For sapphire, the relevant properties were initial density of $3.985 \mathrm{~g} / \mathrm{cc}, \mathrm{c}_{0}$ of
$11.19 \mathrm{~km} / \mathrm{s}$, and $\mathrm{S}$ of 1 [1]. For PMMA, the properties were initial density of $1.1861 \mathrm{~g} / \mathrm{cc}, \mathrm{c}_{0}$ of $2.59 \mathrm{~km} / \mathrm{s}$, and $\mathrm{S}$ of 1.52 [15]. As will be discussed later, this PMMA fit effectively ignores the low pressure $\mathrm{U}_{\mathrm{S}}-\mathrm{u}_{\mathrm{P}}$ nonlinearity [1] that could affect shots 30-32. For fused silica, a polynomial relating stress to material velocity $u_{P}$ was used $\left(\sigma=131.7 * u_{P}-73.61 * u_{P}^{2}+99.47 u_{P}^{3}-41.63 u_{P}^{4}\right) \quad[1]$. The resulting values for the Hugoniot points for Ultem are given in Table 1, and these data are shown as shock velocity $U_{S}$ versus particle velocity $u_{P}$ in Fig. 6 . The PMMA window error bars are larger than the error bars for the other setups due to experimental difficulties with reflections from the free surface of the window. Due to these relatively large uncertainties in the PMMA Hugoniot data, and also because the PMMA used in this study is of a pedigree not characterized in the shock literature, only the data from sapphire and fused silica Hugoniot experiments are used to calculate the Ultem $U_{S}-u_{P}$ Hugoniot fit shown. These experimental difficulties did not influence the release portion of the profile, and so although the Hugoniot points 
Fig. 3 P- $\mathrm{u}_{\mathrm{P}}$ and corresponding Lagrangian $\mathrm{x}-\mathrm{t}$ plot illustrating impedance matching method for window experiments, where a thin Ultem plate impacts a thick sapphire target at $1.287 \mathrm{~km} / \mathrm{s}$.

Note that the Ultem will separate from the sapphire as it unloads. The unloading states prior to separation are not shown (Color figure online)
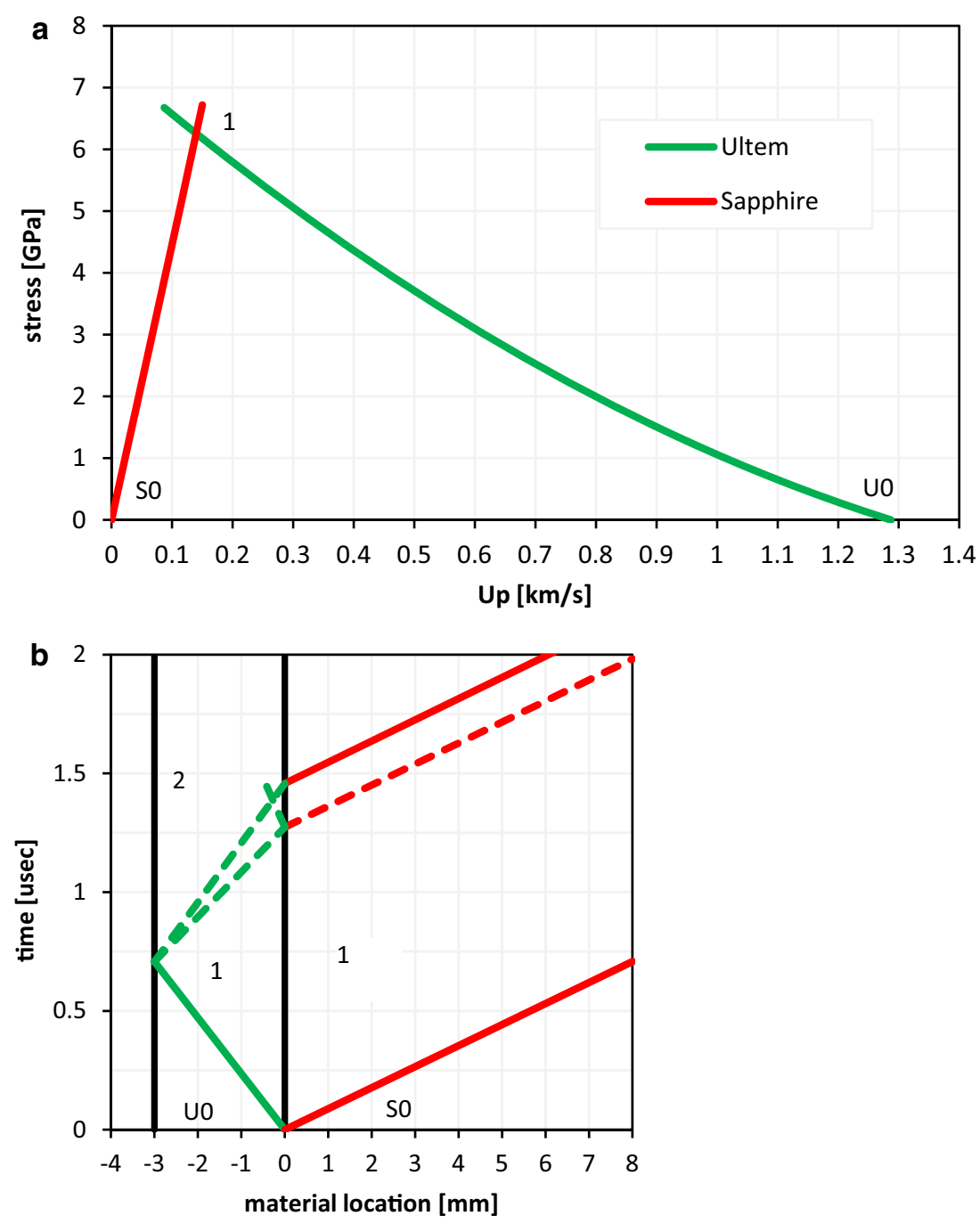

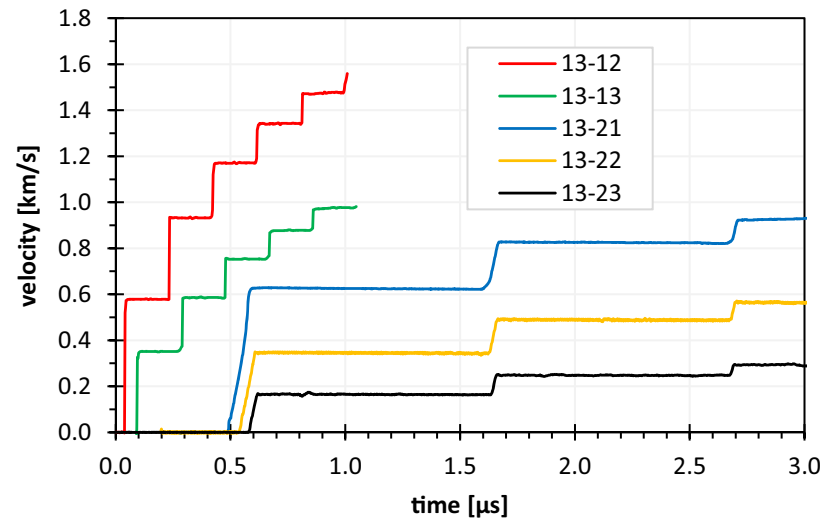

Fig. 4 PDV free-surface velocity traces from the plate-push experiments. The time for each trace is arbitrarily shifted for clarity. As expected, shocks are observed in the sapphire experiments, and ramps in the fused silica experiments (Color figure online)

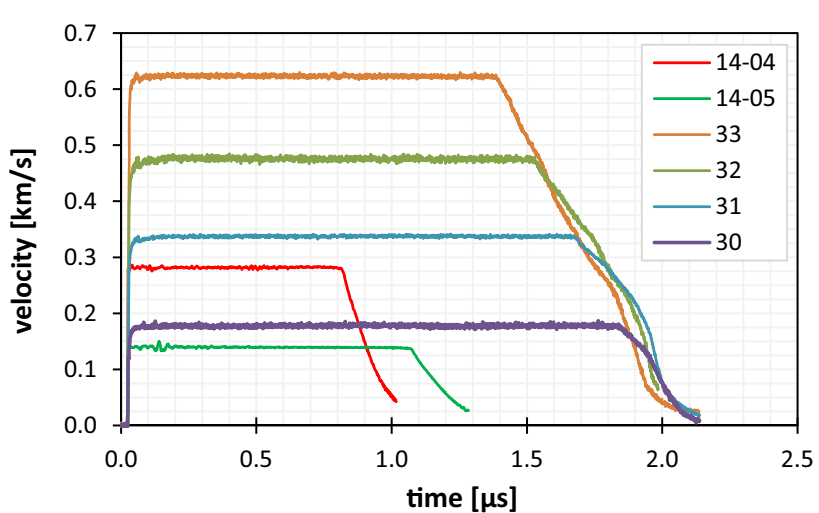

Fig. 5 PDV (for sapphire shots) and VISAR (for PMMA shots) traces from the window experiments, capturing the velocity of the Ultem-window interface. The traces have been corrected for index of refraction effects. Only the four lowest pressure PMMA shots are shown for clarity (Color figure online) 
Fig. $6 \mathrm{U}_{\mathrm{S}}-\mathrm{u}_{\mathrm{P}}$ Hugoniot data for Ultem. The trendline shown in dashed green for the shock velocity, $\mathrm{U}_{\mathrm{S}}=2.42+1.61 * \mathrm{u}_{\mathrm{P}}$, is the linear fit through the experimental Hugoniot data (neglecting the PMMA window data). The trendline shown in dotted grey

$\left(\mathrm{U}_{\mathrm{R}}=2.42+2.65 \mathrm{u}_{\mathrm{P}}{ }^{\wedge} 0.75\right)$ is an estimate of the longitudinal release velocities, plotted here as Eulerian disturbance velocities. Ambient longitudinal and bulk soundspeeds, $\mathrm{c}_{\mathrm{L}}$ and $\mathrm{c}_{\mathrm{B}}$, obtained ultrasonically, are also plotted on the ordinate. Error bars for the sapphire shots are similar in size to the data markers (Color figure online)

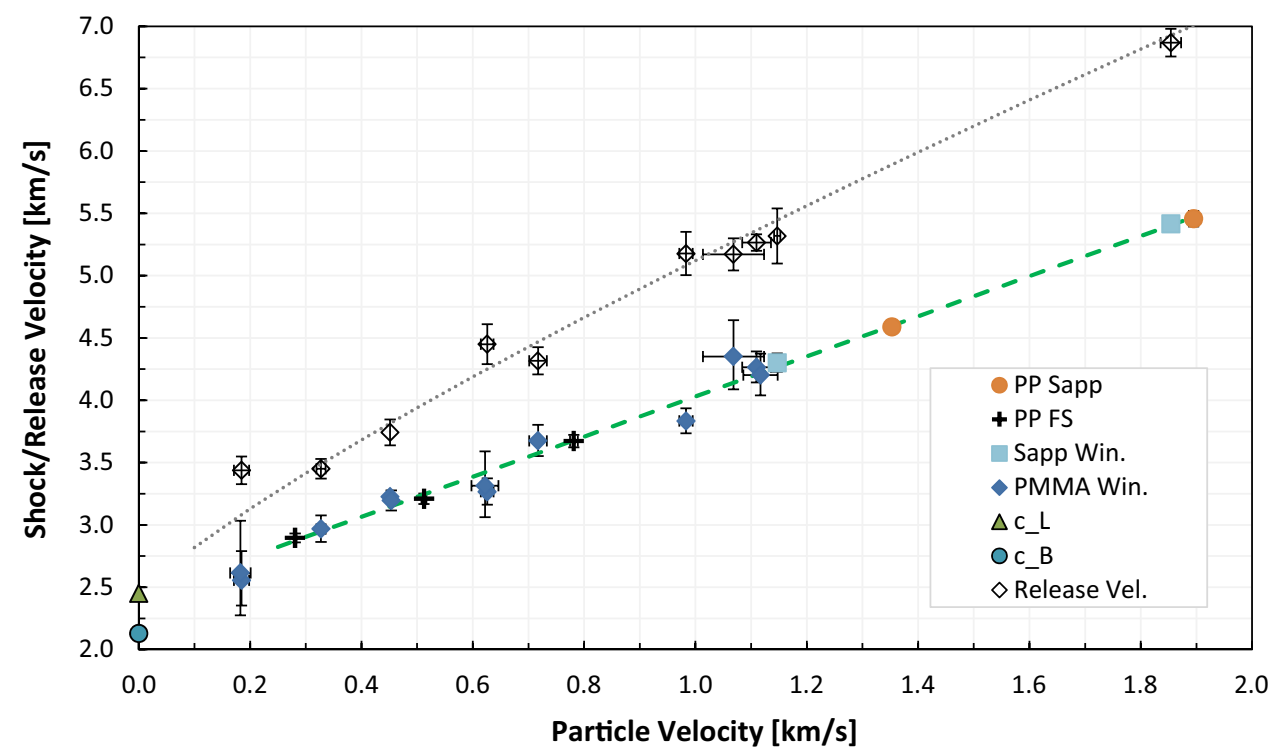

are not used in the Hugoniot fit, we do use the profiles from the PMMA window experiments for quantitative strength measurements discussed later.

Using the velocity traces from the window configuration shown in Fig. 5, together with the sample thicknesses ( $\sim 2.8 \mathrm{~mm}$ ), the leading edge of release wave velocities (sound speeds) for Ultem in the shocked state can be determined. Therefore, Fig. 6 also shows release velocity values obtained from the window experiments described above, along with a suggested fit to the release velocities.

\section{Unloading}

\section{Plate-Push Hysteresis}

For the plate-push data velocity traces shown in Fig. 4, unloading information can be obtained by examining the ring-up of the target plate, since the steps of increasing free surface velocity (after the first step) correspond to unloading steps in the Ultem impactor. This analysis employs the impedance matching technique, and assumes that the target plate unloads along the loading path and is unaffected by repeated load-unload cycles (Fig. 2). Since sapphire and fused silica are within their respective elastic range limits, these assumptions are valid. The analysis also assumes that the unloading is achieved via a simple wave, and assigns each unloading ringup step in the traces in Fig. 4 to an even numbered state on the unloading curve for Ultem, as shown in the impedance matching graphic of Fig. 2. Material properties given previously for sapphire and fused silica were used.

The Ultem volume $V$ at each state was calculated using Eqs. 1 and 2 below, where $d \sigma$ is the stress difference between successive states, $d u_{P}$ is the difference in particle velocity between those same states, and $c_{L}$ is the Lagrangian sound speed for a given particle velocity $u_{P}$. Equation 1 is used to calculate this sound speed, and then Eq. 2 is used to find the change in volume between states $[12,16$, 17].

$d \sigma=\rho_{0} c_{L}\left(u_{P}\right) d u_{P}$

$d V=V_{0} \frac{d u_{P}}{c_{L}\left(u_{P}\right)}$

In this manner, points along the unloading curve in $\mathrm{P}-\mathrm{V}$ space, originating from a corresponding Hugoniot point, are calculated for each of the plate-push experiments. The resulting data is shown in Fig. 7, along with the Hugoniot for reference. Figure 8 shows similar data, but with the stress plotted as deviation from the Hugoniot to highlight the difference in the unloading points and the Hugoniot. The unloading points from the highest pressure plate-push experiment, at $13 \mathrm{GPa}$, lie above the Hugoniot. The unloading points from the intermediate shots at 3-8 GPa (13-13 and 13-21) are below the Hugoniot. The data from the lowest pressure experiments, at 1-2 GPa (13-22 and 13-23), lie essentially on the Hugoniot curve. Figure 9 illustrates the method by which the data in Fig. 8 was reduced to generate the dashed "fit" line.

\section{Window Hysteresis}

For the window experiments, continuous unloading profiles can be obtained by utilizing the latter portion of the profiles from the shock-release window experiments shown in 
Fig. 7 Unloading data for Ultem from the plate push experiments, shown with the same Hugoniot as in Fig. 6. Also, the unloading paths from the two window experiments are shown. It is apparent that the unloading path closely approximates the Hugoniot. The ambient specific volume is also shown, corresponding to the density reported earlier. Error bars are within the data markers. For clarity, the PMMA-window data is not shown (Color figure online)

Fig. 8 Figure 7 data, but plotted as the pressure deviation from the Hugoniot and including the PMMA shot data. The data have been adjusted so that the initial points lie exactly on the Hugoniot. The red dashed line represents the average hysteresis, which indicates a strengthening with increasing shock stress (Color figure online)
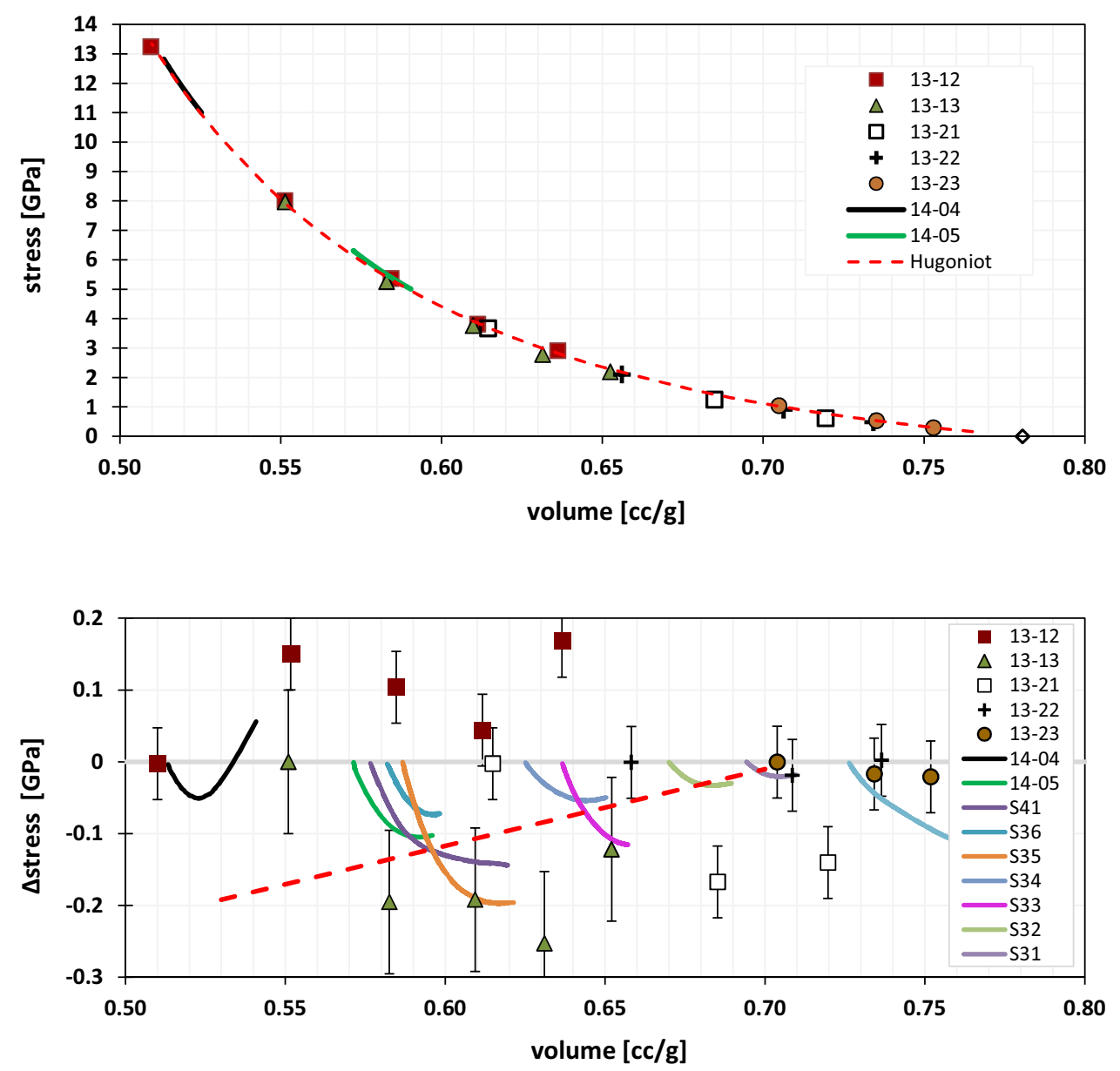

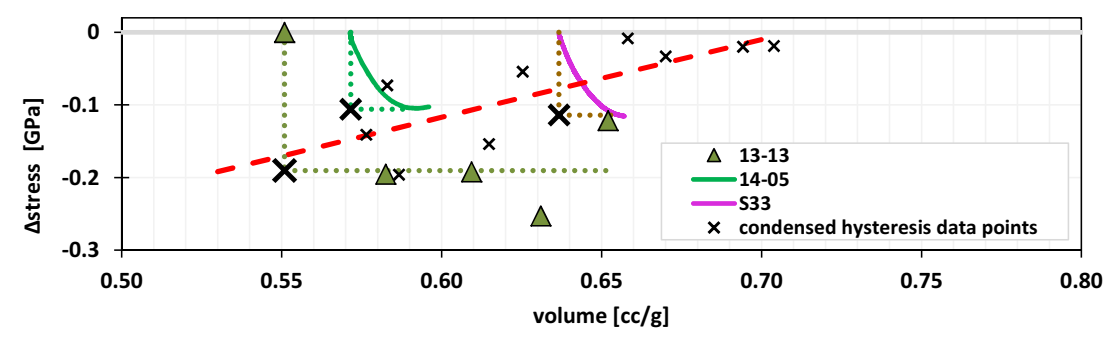

Fig. 9 Illustration of how the dashed fit line in Fig. 8 is constructed, using experiment $13-13$ to illustrate the construction of a data point for a plate-push experiment (error bars removed for clarity), and experiments 14-05 and S33 to illustrate for the window experiments. The data point for each of the three example shots are shown as a

Fig. 5. This latter portion of the interface velocity history contains information about the unloading of the Ultem. The corresponding stress-volume unloading profiles are calculated using the equations already presented. The difference between this analysis of the window data and the previously outlined ring-down analysis of the plate-push data is that in this analysis the unloading states are much closer together. This is due to the fact that the equations are computed for each point in the trace, rather than at each "step" in the trace. large $x$. The data for each of the remaining shots (excluding the highest and lowest pressure shots), constructed in a similar manner, is shown as a small $x$. The dashed line is then fit to the collection of data (Color figure online)

However, the calculation for the window experiments is complicated by the window. A technique is employed which uses Eqs. 1 and 2, along with the Mie-Gruneisen EOS for the window material and the impedance matching technique to calculate the unloading curve of the Ultem. The analysis does not correct for disturbances to the release fan caused by the impedance mismatch at the sapphire window interface, which in this case will cause the latetime Ultem sound speeds to appear slightly lower than they actually are (this effect is essentially absent in the PMMA 
window shots). These errors will accumulate and cause the unloading path to appear to be less stiff. However, simulations run on CTH verified the effect to be small, and so the paths obtained by this method on are also shown in Fig. 7, and the deviation from the Hugoniot is shown in Fig. 8 to accentuate the hysteresis between the Hugoniot and the unloading path. Figure 9 illustrates the method by which the unloading curves in Fig. 8 were interpreted to generate individual data points that are fit by the red dashed line. From Fig. 8, in general the unloading path from the window experiments is below the Hugoniot, as expected and in reasonable agreement with the plate-push data. The unloading path from shot 14-04 dips below the Hugoniot and then rises back above the Hugoniot. This indicates elastic unloading that initially brings the unloading curve below the Hugoniot, followed by isentropic release from elevated temperature that brings the unloading curve back above the Hugoniot. Although this competition between the strength and temperature also occurs at low pressure, the thermal energy deposited into the material in shot 14-04 (13 GPa) is roughly three times that of shot 14-05 (6 $\mathrm{GPa}$ ), and so the resulting dilation is more apparent. For example, using the method of Ref [18], and estimated properties of specific heat $\left(1.5 \mathrm{Jg}^{-1} \mathrm{~K}^{-1}\right)$ and Gruneisen parameter (0.2), the temperature rise in shot $14-04$ is $547 \mathrm{~K}$, versus $187 \mathrm{~K}$ in shot $14-05$. This phenomenon is described in more detail later. The other notable observation in Fig. 8 is the unloading of the lowest pressure window experiment, shot 30 , which is also discussed later. Excepting the highest and lowest pressure shots, agreement is good between the plate-push and window techniques.

\section{Strain-Wavespeed from Window Experiments}

To further estimate the shear stress $\tau$ in the shocked state we applied the technique of Asay and Chhabildas [7] to the unloading data obtained in the window experiments. Although the technique was developed for metals and applied to high strength ceramics that exhibit distinct quasi elastic-plastic behavior rather than polymers where clearly defined elastic-plastic effects are not visible, we apply it here to get another estimate of the magnitude of the shear stress. The technique employs continuous unloading data similar to that shown for the two window experiments in Fig. 7, except that a plot of release velocity versus strain is produced, as shown for several window experiments in Fig. 10. Equation 3 [7] was then applied to determine $2 \tau$.

$2 \tau=-\frac{3}{4} \rho_{0} \int_{\varepsilon_{B}}^{\varepsilon_{1}}\left(c^{2}-c_{B}^{2}\right) d \varepsilon$

where $c$ is measured Lagrangian release velocity, $c_{B}$ is Lagrangian bulk release velocity, $\varepsilon_{1}$ is strain at the initial Hugoniot state, and $\varepsilon_{\mathrm{B}}$ is strain at the transition to bulk release velocity. The initial strain for the integration $\varepsilon_{B}$ was the highest strain at which a break in the slope was observed, and $\varepsilon_{1}$ was the strain at the Hugoniot state from which the material was unloaded. Figure 10 graphically illustrates Eq. 3: the area between $c$ and $c_{B}$ is related to the integral. As shown in Fig. 10, the upper and lower extrapolated bounds represent uncertainty in the location of the slope break, or "kink". This kink is where the transition from elastic to bulk behavior occurs. Although in the elastic-plastic formulation the kink should be the first slope decrease along the unloading path, in some instances two breaks were observed. In these cases the low and high bounds were set to the first and second kink, respectively (for example, see shots 32 and 41 in the data of Fig. 10). As a check of the internal consistency of our results, we can calculate the stress corresponding to the kink in each trace of Fig. 10, and then calculate the corresponding theoretical bulk sound speed from the Hugoniot fit of Fig. 6 and Eq. 4. The resulting theoretical bulk sound speeds are shown as the horizontal dotted lines in Fig. 10, and it is seen that agreement is reasonable in that the resulting bulk wavespeed lies within the low and high bound (except for the lowest pressure experiment which is not surprising since it is presumed viscoelastic).

$c_{R, L a g}^{2}=\frac{(1+S \varepsilon)}{(1-S \varepsilon)^{3}} c_{0}^{2}$

The resulting values are shown in Fig. 11. The lowest data point $(2 \tau=0.01 \mathrm{GPa})$ is likely due to the material being very close to its viscoelastic limit, and as mentioned before is also the only dataset where the identified kink did not agree with the corresponding theoretical bulk wavespeed. At such low compression at $0.6 \mathrm{GPa}$, we infer that the viscoelasticity of the material either causes the Hugoniot begin to deviate from the linear $\mathrm{U}_{\mathrm{S}}-\mathrm{u}_{\mathrm{P}}$ response we report (similar to behavior reported in PMMA [1] ) or causes the elastic and bulk waves to become too indistinct for our analysis to be valid. Thus neglecting the lowest data point, the material displays $2 \tau$ of $\sim 0.06-0.13 \mathrm{GPa}$ over a shock stress range of 1-13 GPa. For ease of comparison, the dashed line from the hysteresis analysis of Fig. 8, transformed into stress- $2 \tau$ coordinates, is also shown in Fig. 11 as a dotted line.

\section{Discussion}

\section{Strength}

There are many indicators of strength in shocked polymers, despite the lack of a distinct elastic precursor as observed 
Fig. 10 Representative plots of wavespeed versus strain, as calculated from the unloading portion of window experiments $30,32,34,41$, and 14-04. Also shown are the $c_{B}$ extrapolations, as well as the estimated upper and lower bounds used for the error bars in Fig. 11. The theoretical bulk wavespeed $\mathrm{c}_{\mathrm{R}}$ (dotted line) is calculated from Eq. 4 using the stress at the "kink" to find the strain. It is observed that there is reasonable agreement between the calculated $c_{R}$ and the identified kink except in shot 30 (Color figure online)

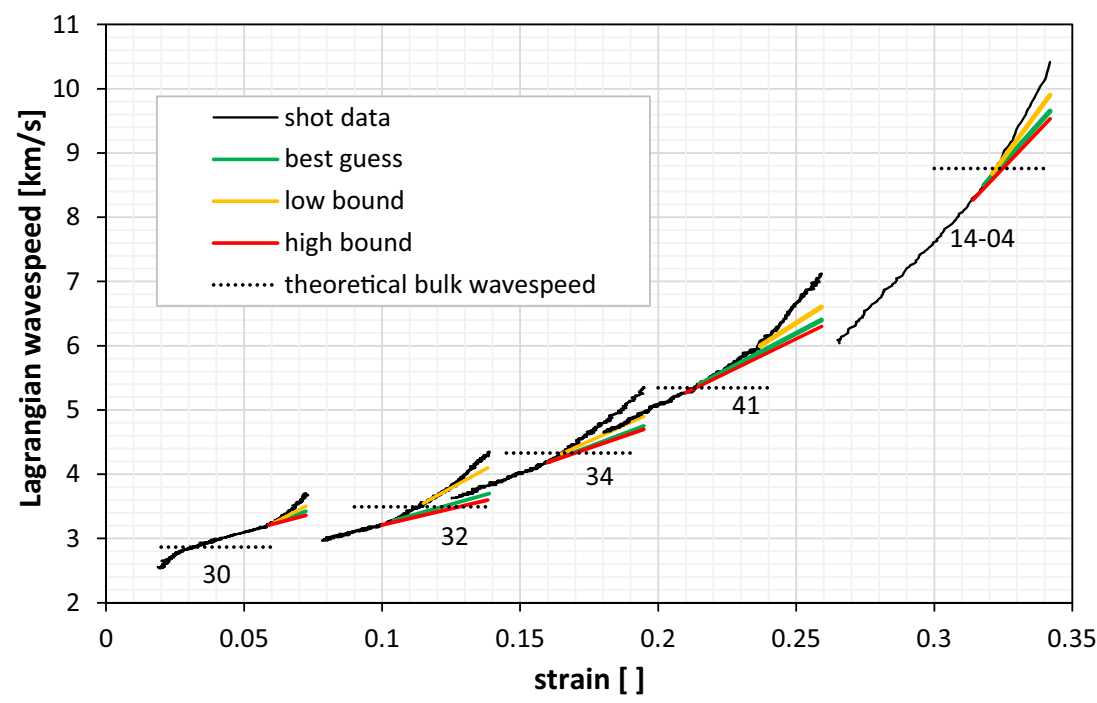

in metals and ceramics. The loading and unloading data of Fig. 7 generally indicates a material that unloads along a P-V path slightly under the Hugoniot loading "path", by an amount ranging from zero to $0.22 \mathrm{GPa}$. Hysteresis in this stress-volume response caused by strength and yield should generally cause the volume upon unloading to be less than the volume upon loading as we observe, and using the von-Mises yield criterion the offset between the loading and unloading paths should be $8 / 3 \tau$. Therefore, a shear strength $\tau$ increasing to $0.08 \mathrm{GPa}$ at $\sim 13 \mathrm{GPa}$ shock stress is implied from Fig. 8.

As noted previously, the exceptions to this in our results are the highest and lowest pressure points. The highest pressure sapphire window shot (shot 14-04) using the hysteresis analysis in Fig. 8 indicates a dip to $0.05 \mathrm{GPa}$ $(\tau=0.02 \mathrm{GPa})$ and then an unexpected rise, while the analysis of the same shot data using the strain-wavespeed analysis of Fig. 10 indicates $\tau=0.07 \mathrm{GPa}$. This was initially surprising since both analyses use the same underlying observation of elevated sound speeds and the same input data. The phenomenon is due to a combination of the thermal dilation effect on the unloading path and the very gradual transition from elevated "elastic" wavespeed to bulk soundspeed. For example, in shot 14-04, the Hugoniot stress is $12.9 \mathrm{GPa}$, but the stress in the material when it transitions from elastic to bulk wavespeed is only 10.1 GPa. If the thermal energy deposited into the material is sufficient, the transition from elastic wavespeed to bulk wavespeed can occur at a point well beyond the apparent maximum hysteresis seen in an analysis such as that of Fig. 8. In fact, for shot 14-04, the point where the wavespeed kink occurs is well beyond the point of maximum hysteresis, and is actually above the Hugoniot. However, in shot 14-05 (sapphire window at $6.2 \mathrm{GPa}$ ), these two points (point of max. hysteresis and point of kink in wavespeed) are much closer, and both are below the Hugoniot. For the highest pressure plate-push experiment, similar arguments apply. The initial unloading is not captured because the first released state interrogated by the sapphire plate is sufficiently far below the Hugoniot state that the thermal dilation has obscured the strength. In fact, it is observed in Fig. 8 that the window unloading from $\sim 13 \mathrm{GPa}$ (shot 14-04) approaches the first unloading state captured in 13-12. The lowest pressure point is discussed in the following paragraph.

From the separate analysis of Figs. 10 and 11 on the window experiments, $2 \tau$ is on the order of $0.06-0.13 \mathrm{GPa}$ yielding a shear strength $\tau$ of $0.03-0.07 \mathrm{GPa}$ as shock stress increases. Again, the lowest pressure experiment at $0.6 \mathrm{GPa}$ is the exception, showing virtually no strength due to viscoelastic behavior. It is notable that this lowest pressure point (shot 30 at $0.6 \mathrm{GPa}$ ) is the exception in both our analysis, and also why it displays such a discrepancy between strength inferred from the two analysis. In the wavespeed versus strain analysis given by Eq. 3, the differences are not dependent on the absolute magnitude of the Hugoniot itself. The strength estimate only depends on the wavespeed/strain continuous release profile under consideration. However, in the window hysteresis analysis, the strength is based on the difference between the release profile and the average Hugoniot determined by the $U_{S}-u_{P}$ fit of all experiments. Experiments on PMMA [1] suggest that the $\mathrm{U}_{\mathrm{S}}-\mathrm{u}_{\mathrm{P}}$ behavior is not linear at low pressures $(<2 \mathrm{GPa})$; it was found that the $\mathrm{U}_{\mathrm{S}}-\mathrm{u}_{\mathrm{P}}$ data were above those extrapolated from high pressures due to viscoelasticity. We believe a similar phenomenon is occurring with Ultem, steepening the initial $\mathrm{P}-\mathrm{V}$ Hugoniot, and so the steeper release we observe in the hysteresis analysis on shot 30 is due to this phenomenon. Therefore, our anomalous hysteresis in shot 30 is likely due to Ultem 
Fig. 11 Shear strength of Ultem as a function of Hugoniot stress, calculated using the technique of ref [7]. Again neglecting the lowest data point, the data indicate an upward trend (red dashed line). The dotted line represents the fit from the hysteresis analysis shown in Fig. 8 (where the $\Delta$ stress is assumed to be $8 / 3 \tau)$, transformed into the coordinates shown here (Color figure online)

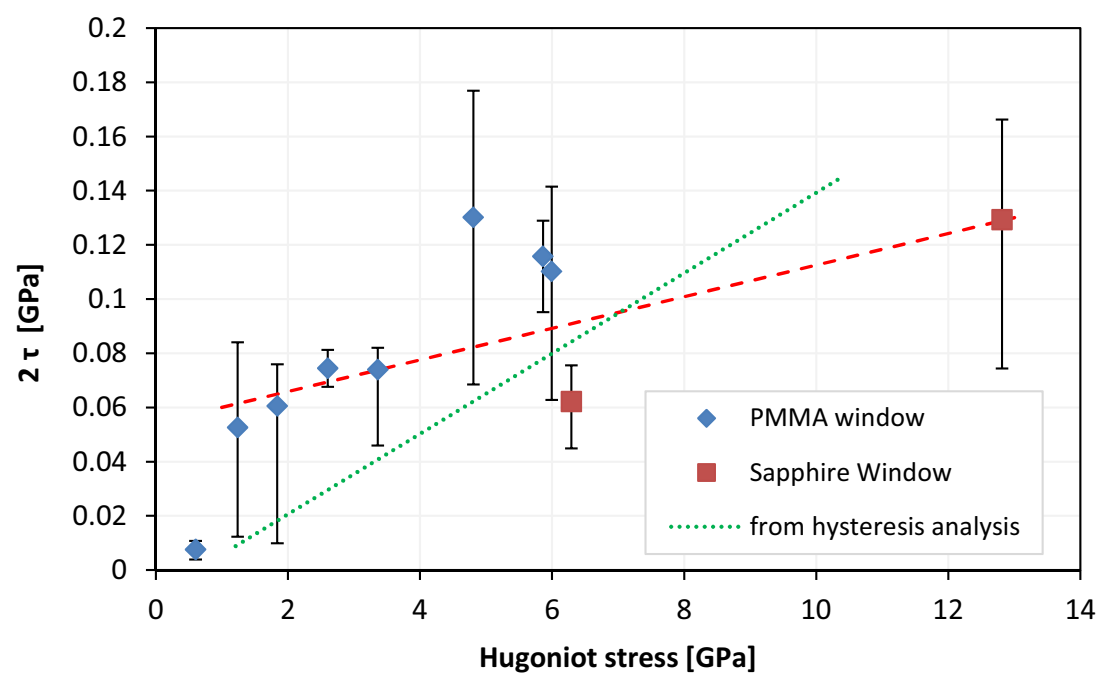

displaying viscoelastic behavior similar to PMMA, with elevated shock velocities at very low $u_{P}$. It may be noted that if this hypothesis were true, that our Hugoniot points in Fig. 6 for shot 30 should be above the extrapolated line, rather than below. Indeed, if we use the nonlinear PMMA Hugoniot data of Barker and Hollenbach [1] for shot 30 in the impedance matching calculation, we do find the Ultem points for shot 30 (as well as 31 and 32) are shifted to above the extrapolated line (to $\mathrm{U}_{\mathrm{S}}=2.83 \mathrm{~km} / \mathrm{s}$ for shot 30). However, we have elected not to use the nonlinear PMMA Hugoniot due to uncertainty regarding the differences in our PMMA versus that measured by Barker and Hollenbach [1].

In light of these considerations, the findings of the two shear strength analyses are in remarkably good agreementboth show $\tau$ increasing from approximately $0.03 \mathrm{GPa}$ to $0.08 \mathrm{GPa}$ as the shock stress increases from 1 to $13 \mathrm{GPa}$. Furthermore, this comparison demonstrates the importance of capturing continuous release (as opposed to step-wise) data for low-strength materials when shocked to sufficiently high levels, although in principal the hysteresis could also be captured using plate-push configurations with very high impedance plates (such as diamond) so that the release steps would be smaller.

\section{Prediction of Loss of Strength}

Finally, the disturbance velocities reported in Fig. 6 can be compared with the theoretical bulk sound speeds of the Hugoniot in order to again infer the presence of strength, and also predict when the material will lose strength. This is most easily accomplished in $\sigma-V$ space, since the disturbance velocity at pressure is simply related to the bulk modulus, and therefore $\mathrm{d} \sigma / \mathrm{d} V$. If the material behaves hydrodynamically (no strength), the measured disturbance velocity will approach the local slope of the Hugoniot. Alternatively, if the release is steeper than the Hugoniot, it is an indication of strength. Following Bourne et al. [19] we can compute the expected ratio of the sound speed to the shock velocity, assuming hydrodynamic behavior based on the applicability of the empirical $\mathrm{U}_{\mathrm{S}}=\mathrm{c}_{0}+\mathrm{S}^{*} \mathrm{u}_{\mathrm{P}}$ relationship. The resulting ratio, as a function of volumetric strain $\varepsilon$, is

$\frac{c_{R}^{2}}{U_{S}^{2}}=\frac{c_{0}^{2}(1-\varepsilon)^{2}(1+S \varepsilon)}{(1-S \varepsilon)^{3}\left[c_{0}+S\left(\frac{c_{0} \varepsilon}{1-S \varepsilon}\right)\right]^{2}}$

An observed ratio of $c_{R} / U_{S}$ that is greater than this hydrodynamic ratio indicates strength. Although at low pressures it is known that the intercept $c_{0}$ does not represent the true ambient bulk behavior, our previously discussed results (in Fig. 10) indicate that it is representative above $1 \mathrm{GPa}$. Therefore, Fig. 12 shows such normalized release velocity data for Ultem, as well as for the other polymers with available sound speed data in the shocked state.

In the work of Bourne et al. [19], the $c_{R} / U_{S}$ ratio was plotted with respect to $\mathrm{u}_{\mathrm{P}}$, and the observation was made that the data intersected the hydrodynamically predicted ratio based on Eq. 5 at $\mathrm{u}_{\mathrm{P}}=0.7 \mathrm{~km} / \mathrm{s}$ for the three materials investigated in that work (PTFE, PVDF, and PE). However, when plotting all the materials shown in Fig. 12 against $u_{P}$ (not shown), it is observed that the intersection at $\mathrm{u}_{\mathrm{P}}=0.7 \mathrm{~km} / \mathrm{s}$ is not generally true, and does not work for many of the other materials shown. In Fig. 12 we have plotted the ratio against the dimensionless measure of deformation, strain, and again we see that there is no general rule for when the observed value approaches the hydrodynamic value. For all the materials except two of the fluorinated polymers, PTFE and Viton $\mathrm{B}$, the observed ratio is $\sim 1.2$, and so the strongest indicator of where the 


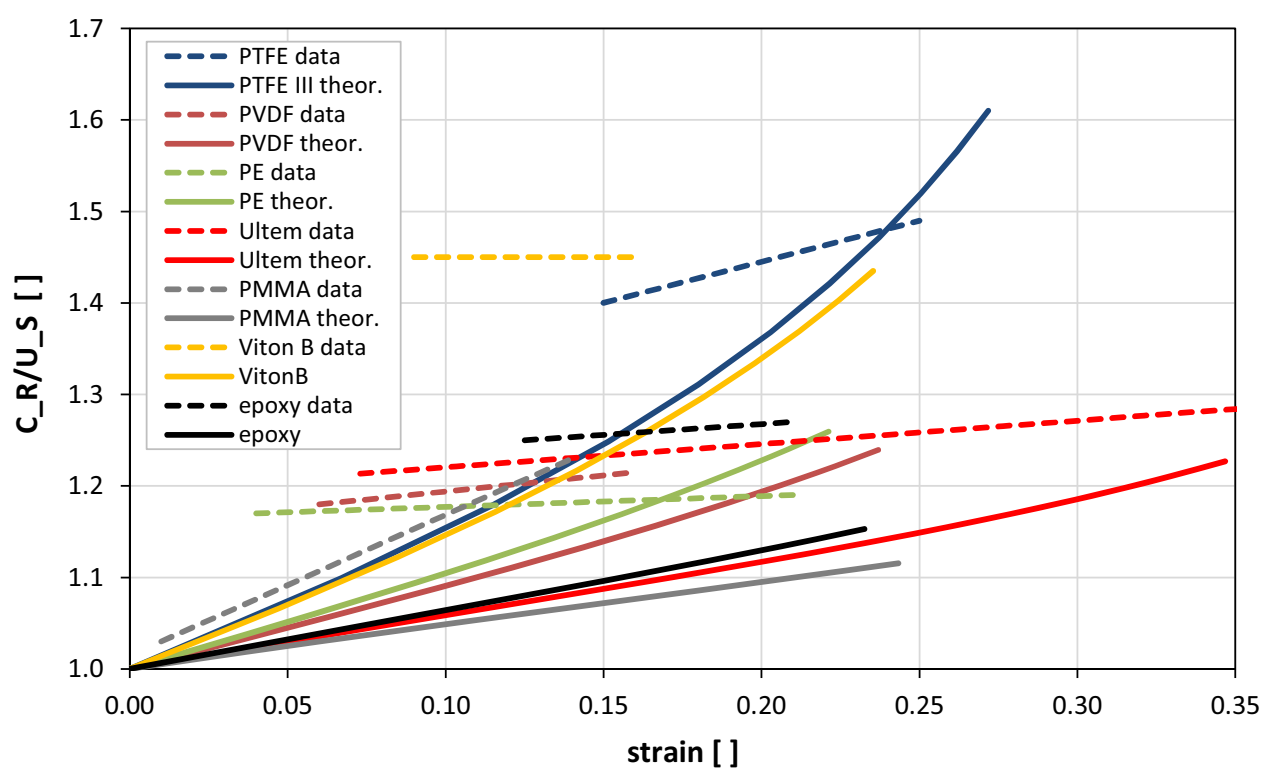

Fig. 12 Ratio of the Eulerian release velocity $c_{R}$ to the shock velocity $\mathrm{U}_{\mathrm{S}}$ versus strain $\varepsilon$. The dashed lines are linear fits to data from the literature (individual points not shown for clarity), and the solid curves are the hydrodynamic (zero strength) ratios computed from Eq. 5. PTFE, PVDF, and PE data from [19], PMMA data from [1], Viton B data from [20], epoxy data from [21]. For the Ultem data

crossover will occur is simply when the hydrodynamic ratio reaches that range. For the formulation of Eq. 5, this crossover will occur at low strains $(\varepsilon<0.25)$ for high $\mathrm{S}$, low $\mathrm{c}_{0}$ materials, and at high strains $(\varepsilon>0.3)$ when $\mathrm{S}$ is small and $\mathrm{c}_{0}$ is large. It is interesting to note that the four materials in Fig. 12 with crossovers at low strain, in addition to high $\mathrm{S}$, low $\mathrm{c}_{0}$ values, are semi-crystalline polymers. On the other hand, the materials with low $\mathrm{S}$, high $\mathrm{c}_{0}$ parameters are amorphous polymers. This may indicate that regardless of the $c_{R} / U_{S}$ ratio, crossover (and therefore loss of strength) occurs at lower strains in partially crystalline systems.

For the PMMA data in Fig. 12, it is apparent that if the data is extrapolated, it will never intersect the hydrodynamic ratio. However, it has been shown for polyethylene that this ratio initially increases and then levels off as strain or $u_{P}$ increases [22], and it is expected that PMMA will display this same behavior and that crossover will occur at high strain. This analysis is in good agreement with the strength results presented earlier where we reported strength even at $13 \mathrm{GPa}$, since from Fig. 12, even at our highest pressure data, we have not yet reached the crossover point for Ultem. Extrapolating our data, we predict that Ultem will continue to indicate strength up to a shock strain of 0.4 , although the strength may begin to decrease as the crossover is approached due to thermal softening. However, as previously discussed, we do not see evidence (in red), the dashed line corresponds to the dashed line in Fig. 6 for the release velocities. We note that a similar analysis can be performed utilizing Poisson's ratio $v$ : we find that $v$ increases from 0.39 at ambient to 0.46 at $13 \mathrm{GPa}$, showing that $v$ approaches, but does not intersect, 0.5 , just as the dashed Ultem line approaches but does not intersect the solid curve (Color figure online)

of weakening at a shock strain of 0.35 ; if anything, Ultem is still strengthening.

In other work, the lateral gauge technique has also been employed as both embedded manganin $[3,5]$ and embedded electromagnetic gauges [6] to measure the strength of shocked polymers, also assuming an elastic-plastic solid. In those studies, it was generally (but not always) found that the shear stress increased with Hugoniot stress. This is consistent with our findings, as shown in the trendlines of Figs. 8 and 11. However, the values reported elsewhere [3, 5] are $\sim 10 \times$ higher than those found here for Ultem, ranging from $\tau=0.1-0.7 \mathrm{GPa}$. Unfortunately, the study on PMMA [6], which may behave most similarly to Ultem, was at very low pressure and unlikely to extrapolate to the pressures examined here. The higher strength values reported using embedded manganin gauges may partially be explained by recent work revising the calibration of the lateral manganin gauges, which will result in lower calculated strength values [23].

Overall, a picture is presented indicating that Ultem displays shear strength $\tau$ in the shocked state of $\sim 0.05 \mathrm{GPa}$. Specifically, we find that $\tau$ increases with shock stress, from $\tau=0.03 \mathrm{GPa}$ when shocked to $1 \mathrm{GPa}$, to $\tau=0.08 \mathrm{GPa}$ when shocked to $13 \mathrm{GPa}$. While the various analyses done here supporting this conclusion are not completely independent, and the values found are near the lower limit of what we can confidently resolve, we 
nonetheless conclude that we can see evidence of strength and strengthening in these experiments.

\section{Conclusions}

Parallel-plate impact studies using a single stage powder gun have been performed to investigate the shock and subsequent release behavior of the commercial polyetherimide polymer Ultem. Two different types of setups were used to observe both the loading and unloading behavior. In one, the sample impacted a window, and in the other, the sample impacted a witness plate. As expected, the results from the two methods concerning the loading behavior agreed very well and the resulting Hugoniot was reported. For unloading, this study also demonstrated that the witness plate method, though not recording the continuous unloading path of the sample, agrees with the continuous method, although it may not record strength at higher pressures due to the effects of thermal dilation and is subject to inaccuracies in the Hugoniot at low pressure. The unloading data presented were used to build a case that the strength $\tau$ of Ultem increases from $\sim 0.03-0.08 \mathrm{GPa}$ as shock stress increases from 1-13 GPa. Furthermore, an investigation of the ratio of the release wave velocity to the shock wave velocity indicated that a transition to bulk liquid (no strength) behavior is not achieved until Hugoniot strains exceed 0.35 for amorphous polymers such as Ultem, whereas it is achieved at lower strains for more crystalline polymers. In future work, we would like to apply these techniques to polymers that have been reported in the literature to display higher deviatoric stresses.

Acknowledgments We would like to thank Rick Davis and Adam White for skillful assembly and execution of the experiments, and Don Cunard and Dr. Robert Dorgan for providing both the motivation and the samples used in this study.

\section{References}

1. Barker L, Hollenbach R (1970) Shock wave studies of PMMA, fused silica, and sapphire. J Appl Phys 41:4208-4226

2. Munson D, May R (1971) Dynamically determined high-pressure compressiblities of three epoxy resin systems. J Appl Phys 43(3):962-971

3. Bourne N, Gray G (2005) Dynamic response of binders; teflon, estane, and Kel-F-800. J Appl Phys 98:123503
4. Carter W, Marsh S (1995) Hugoniot equation of state of polymers, Los Alamos National Laboratories

5. Bourne N, Millett J, Brown E, Gray G (2007) Effect of halogenation of the shock properties of semicrystalline thermoplastics. J Appl Phys 102:063510

6. Gupta Y (1980) Determination of the impact response of PMMA using combined compression and shear loading. J Appl Phys 51(10):5352

7. Asay J, Chhabildas L (1981) Determination of the shear strength of shock compressed 6061-T6 aluminum. Shock waves and highstrain-rate phenomena in metals. Plenum press, Albuquerque

8. Neel C, Chhabildas L, Maines W, Johnson M, Davis R, White A. AFRL HP3 facility four years in - final report for WU W09G," AFRL Report\# AFRL-RW-EG-2015-004

9. Strand O, Goosman DR, Martinez C, Whitworth T, Kuhlow W (2006) Compact system for high-speed velocimetry using heterodyne techniques. Rev Sci Instrum 77:083108

10. Barker L, Hollenbach R (1972) Laser interferometer for measuring high velocities of any reflecting surface. J Appl Phys 43(11):4669-4675

11. Forbes JW (2012) Shock wave compression of condensed matter. Springer, Berlin

12. Chhabildas L, Grady D (1983) Dynamic material response of quartz at high strain rates. In: Material Research Society Symposium Proceedings, 1983

13. Jensen B, Holtkamp D, Rigg P, Dolan D (2007) Accuracy limits and window corrections for photon Doppler velocimetry. J Appl Phys 101:013523

14. Chhabildas L, Asay J (1979) Rise-time measurements of shock transitions in aluminum, copper, and steel. J Appl Phys 50(4):2749-2756

15. Marsh SP (1980) LASL shock hugoniot data. University of California Press, Berkley

16. Asay J, Hall C, Knudson M (2001) Recent advances in highpressure equation-of-state capabilities. Shock-wave and highstrain-rate phenomena. Elsiever, Oxford

17. Fowles R, Williams RF (1970) Plane stress wave propagation in solids. J Appl Phys 41(1):360-363

18. Davison L (2008) Fundamentals of shock wave propagation in solids. Springer, Berlin

19. Bourne N, Brown E, Millett J, Gray G (2008) Shock, release and Taylor impact of the semicrystalline thermoplastic polytetrafluoroethylene. J Appl Phys 103:074902

20. Millett J, Bourne N, Gray G (2004) The equation of staet of a fluorinated tripolymer. J Appl Phys 96(10):5500-5504

21. Anderson M, Setchell R, Cox D (1999) Shock and release behavior of filled and unfilled epoxies. In Shock compression of condensed matter 1999, Snowbird, UT

22. Mori Y, Nagayama K (1999) Measurement of the slope of shock velocity-particle velocity hugoniot curve for polymers. In: Shock compression of condensed matter 1999, Snowbird, UT

23. Rosenberg Z, Moshel G (2014) Revisiting the calibration of manganin gauges for lateral stress measurements in shock-loaded solids. J Appl Phys 115:103511 